\title{
CsI Calorimeter and low power PMT base for $\mathrm{K}^{\mathrm{O}} \mathrm{TO}$ experiment
}

\section{Takahiko Masuda* for the J-PARC E14 KO TO Collaboration}

High Energy Physics Group, Department of Physics,

Faculty of Science, Kyoto University

Kitashirakawa Oiwake-cho Sakyo-ku, Kyoto-shi,

Kyoto, Japan 606-8502

E-mail: taka@scphys.kyoto-u.ac.jp

$\mathrm{K}^{\mathrm{O}} \mathrm{TO}$ is the first experiment in the world specialized in observing the decay $K_{L} \rightarrow \pi^{0} v \bar{v}$. The decay is sensitive to new physics scenarios beyond the Standard Model. The most important detector of $\mathrm{K}^{\mathrm{O}} \mathrm{TO}$ is the CsI calorimeter, which measures positions and energies of gammas from $K_{L}$, in order to reconstruct the vertexes of them. There are mainly three requirements for the calorimeter. First, dynamic range for each channel of CsI is more than 3 order of magnitude. Second, single counting rate is about $120 \mathrm{kHz}$ comes from beam intensity. And linearity is required less than $\pm 5 \%$ in order to discriminate the signals and backgrounds. Finally, to suppress the backgrounds, we will put the calorimeter at the vacuum level of $0.1 \mathrm{~Pa}$. So we must reduce its power consumption. That is why we plan to use the Cockcroft-Walton circuit as a high voltage supply for PMTs. It can reduce power down to about $150 \mathrm{~mW}$.

2009 KAON International Conference KAON09,

June 09 - 122009

Tsukuba, Japan

${ }^{*}$ Speaker. 


\section{1. $\mathrm{K}^{\mathrm{O}} \mathrm{TO}$ experiment}

$\mathrm{K}^{\mathrm{O}} \mathrm{TO}$ is the experiment specialized in observing the decay $K_{L} \rightarrow \pi^{0} v \bar{v} . \mathrm{K}^{\mathrm{O}} \mathrm{TO}$ will start from JFY2011 in J-PARC. We plan to observe some $K_{L} \rightarrow \pi^{0} v \bar{v}$ events in three years.

\section{1 $K_{L} \rightarrow \pi^{0} v \bar{v}$}

The branching ratio of $K_{L} \rightarrow \pi^{0} v \bar{v}$ decay predicted by standard model is presented as

$$
\operatorname{Br}\left(K_{L} \rightarrow \pi^{0} v \bar{v}\right)=(2.20 \pm 0.07) \times 10^{-10}\left[\mathfrak{I}\left(V_{t s}^{*} V_{t d}\right) X\left(x_{t}\right)\right]^{2},
$$

and current value is $(2.49 \pm 0.39) \times 10^{-11}$ [1]. Where $X\left(x_{t}\right)=1.464 \pm 0.041$ is Inami-Lim loop function[2]. $x_{t}$ is squared mass ratio of a top quark and a $\mathrm{W}$ boson. Presented as Wolfenstein parameters, $\mathfrak{I}\left(V_{t s}^{*} V_{t d}\right)=A^{2} \lambda^{5} \eta$, so there is the CP violation parameter $\eta$ representing the hight of Unitary triangle. Then, measuring the branching ratio of the decay $K_{L} \rightarrow \pi^{0} v \bar{v}$, we can determine it.

\subsection{CsI calorimeter}

We plan to use CsI calorimeter to measure positions and energies of gammas from the decay $K_{L} \rightarrow \pi^{0} v \bar{v}$, and the $\pi^{0} \rightarrow 2 \gamma$, in order to reconstruct the vertex of $K_{L}$ (Fig. 11). We reuse about 3000 blocks of CsI crystals used in $\mathrm{KTeV}$ experiment for the calorimeter at the Fermi National Accelerator Laboratory. There are $2.5 \times 2.5 \times 50 \mathrm{~cm}$ crystals and $5 \times 5 \times 50 \mathrm{~cm}$ crystals. We also reuse photomultiplier tubes used in $\mathrm{KTeV}$.

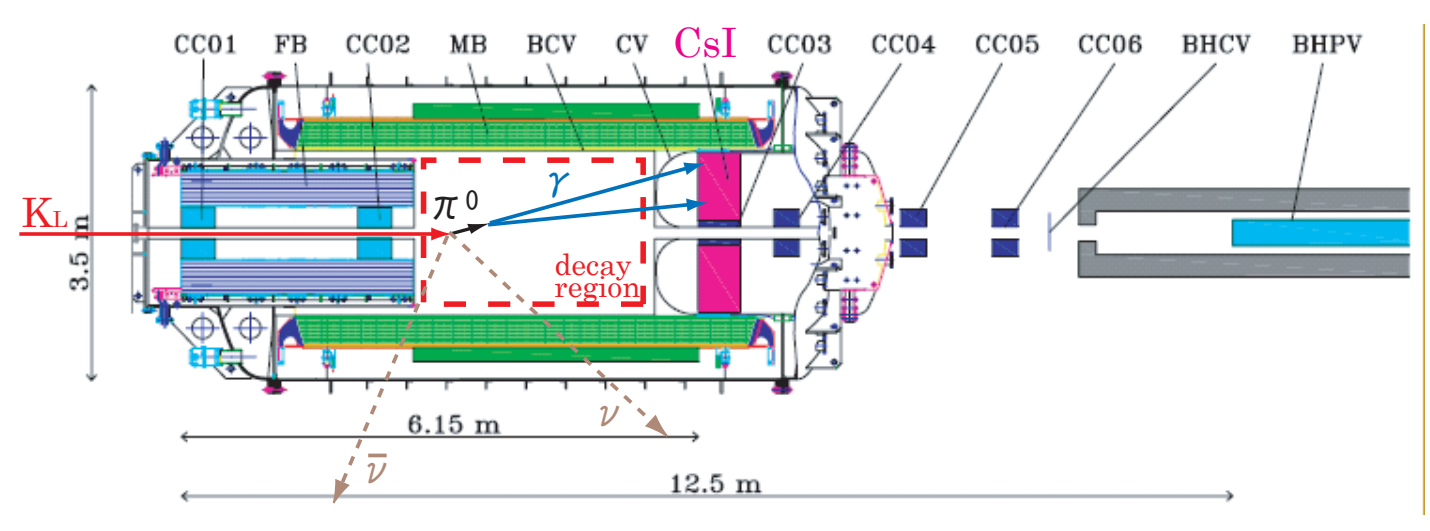

Figure 1: schematic view of detector setup

\section{Requirements for CsI calorimeter}

\subsection{Single counting rate}

The beam used in $\mathrm{K}^{\mathrm{O}} \mathrm{TO}$ experiment will be neutral and sharply collimated pencil beam. All of particles entering into the calorimeter are generated from $K_{L}$ decays. So single counting rate of CsI calorimeter is not so high. Fig. 2 shows the single counting rate of each crystal simulated by Geant 4 . The hit rate is only $120 \mathrm{kHz}$ even in case of highest one. 


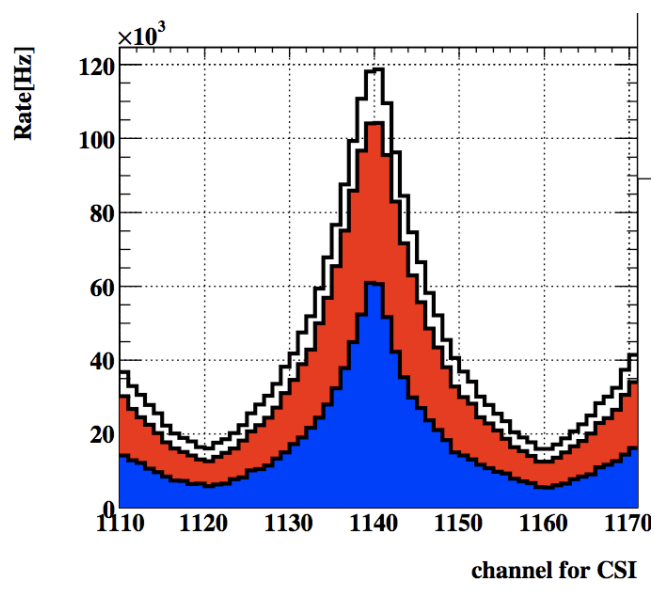

Figure 2: Single counting rate of each crystal. This graph shows only around hottest crystal which are located around beam hole. White histogram is all energy event, red one is over $0.1 \mathrm{MeV}$ event, blue one is over $1 \mathrm{MeV}$ event.

\subsection{Linearity}

Linearity is defined as the deviation of ratio between deposit energy and measured energy like,

$$
\frac{E_{\text {measured }}}{E_{\text {deposit }}},
$$

when $E_{\text {measured }}$ is PMT output signal and $E_{\text {deposit }}$ is real energy deposited in CsI. If the linearity is worse, the number of signal and backgrounds change, because the $\mathrm{K}_{\mathrm{L}}$ vertex and transverse momentum are not correctly reconstructed due to miss measured energy. Injecting into CsI calorimeter, $\gamma$ makes electro magnetic shower and deposits their energy to multiple crystals. In our analysis, we cluster those multiple energy deposits and reconstruct position and energy of $\gamma$. That is why, deviation of measured energy of each crystal changes clustering result and reconstructed vertex. Miss reconstruction of $K_{L}$ causes to mistake other decay modes for background. Fig 3 shows the quantitative estimation about it. According to this simulation result, CsI calorimeter linearity is required less than $\pm 5 \%$.

\subsection{Energy range}

There is a necessity to estimate the energy range of deposit energy for each crystal. Fig. 4 shows maximum deposit energy of one gamma from the decay $K_{L} \rightarrow \pi^{0} v \bar{v}$. Without any cut, deposit energies distribute over $2 \mathrm{GeV}$, but such high energy region is vanished by high energy kinematic cut which is for $K_{L} \rightarrow 2 \gamma$. Finally we decided the energy range upper limit is $1.3 \mathrm{GeV}$. And also CsI calorimeter has a role as a $\gamma$ veto detector, so the energy range lower limit is $1 \mathrm{MeV}$.

\section{Development of low power PMT base}

As noted above, we reuse $\mathrm{KTeV}$ photo multiplier tubes(PMT). But there are some problems. One of them is heat problem. Major $\mathrm{K}^{\mathbf{0}}$ TO detectors will be located in vacuum $(0.1 \mathrm{~Pa})$, because air and beam neutrons reaction makes $\pi^{0}$ which is background source. Then there is heat problem 

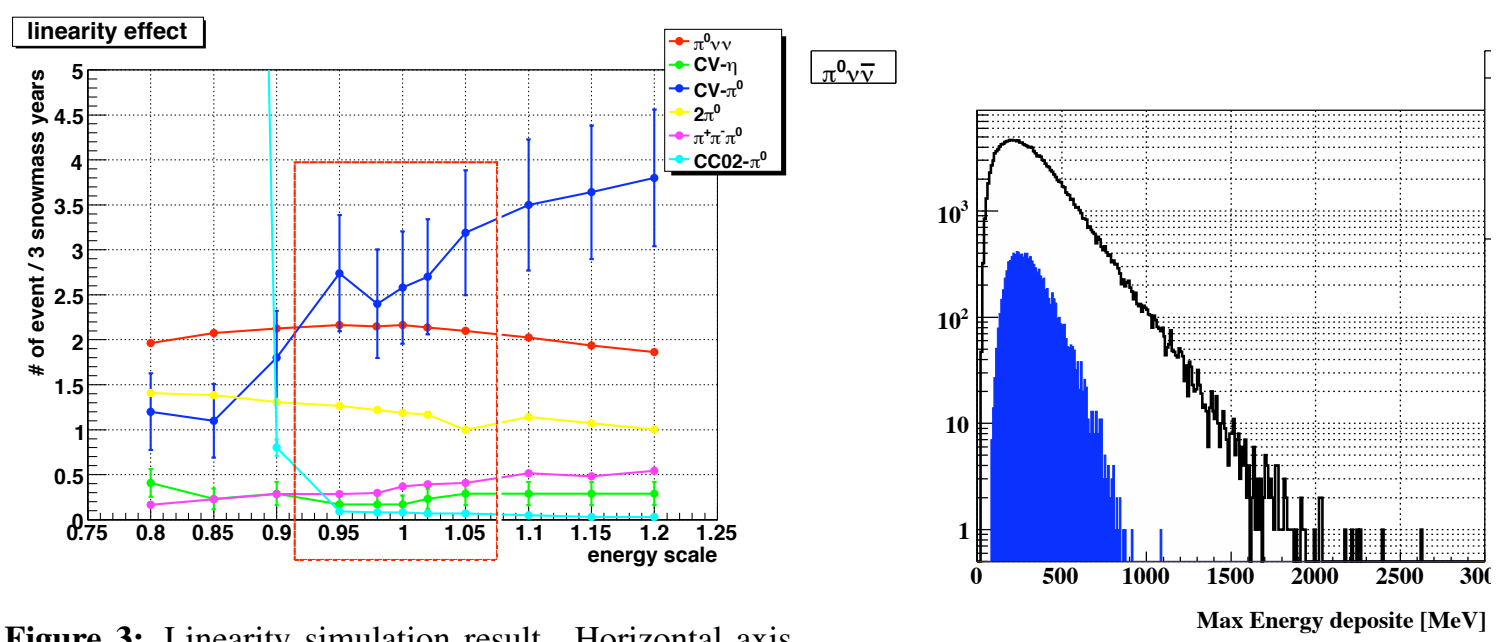

Figure 3: Linearity simulation result. Horizontal axis is defined in Eq. 2.1. Red line is the expected number of signal event with all runs, and other lines are that of Figure 4: The deposit energy distribution from background event. Under 0.95 and over $1.05, \mathrm{CV}-\pi^{0}$ and the decay $K_{L} \rightarrow \pi^{0} v \bar{v}$ CCO2- $\pi^{0}$ event increase.

caused by operation in vacuum using normal resistor-divided base. We must reduce PMT base power consumption to suppress heat. Other problem is quite small gain of KTeV PMT. The gain of $\mathrm{KTeV}$ PMT is only 5000, because average energy deposit in $\mathrm{KTeV}$ experiment was about $80 \mathrm{GeV}$. In contrast, maximum deposit energy of $\mathrm{K}^{\mathbf{0}} \mathrm{TO}$ is only around $2 \mathrm{GeV}$, so we had to increase its gain. That is why I planed to use Cockcroft-Walton circuit[4] as PMT base, and designed low power consumption preamp.

\subsection{CW circuit}

Cockcroft-Walton circuit consisting of diodes and capacitors bridge generates high voltage with low voltage supply. Using this circuit, we could reduce power consumption from $700 \mathrm{~mW} / \mathrm{ch}$ to $150 \mathrm{~mW} / \mathrm{ch}$, that value is reasonable for cooling. In this experiment, we must reduce noise level in order to get 3 order dynamic range, so RC filters are attached to $\mathrm{CW}$ circuit between every dynode pins and diodes (Fig. 57).

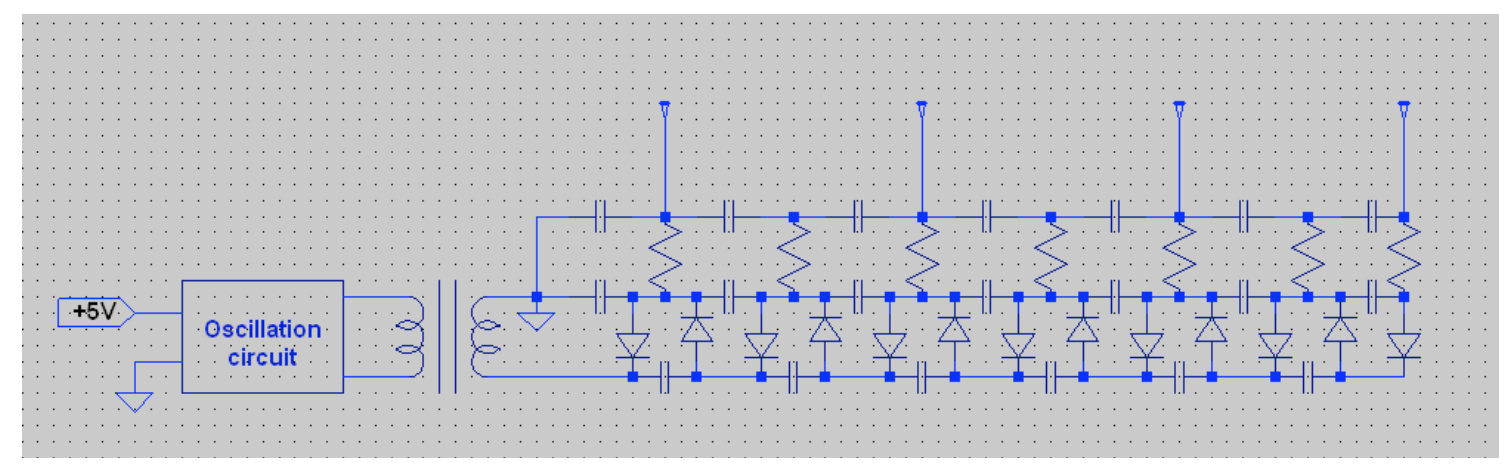

Figure 5: CW circuit diagram. There are RC filters between $\mathrm{CW}$ circuit and dynodes. 


\subsection{Preamp}

The gain of KTeV PMT was about 5000. It is too low to detect $1 \mathrm{MeV}$ signal. So I designed a high-speed, low power consumption and low noise preamp to increase PMT signal enough to detect $1 \mathrm{MeV}$ signal.

\section{Conclusion}

$\mathrm{K}^{\mathrm{O}} \mathrm{TO}$ is the first experiment in the world specialized in observing the decay $K_{L} \rightarrow \pi^{0} v \bar{v}$. The most important detector is the CsI calorimeter, which measures the positions and energies of gammas from $K_{L}$, in order to reconstruct the vertex of it.

There are several requirements for CsI Calorimeter in order to observe signal event with good $\mathrm{S} / \mathrm{N}$ ratio. We designed new PMT bases which suffices them adopting filtered CW circuit and highspeed and low noise preamp. Table 1 shows major specifications and performance of our new PMT base. This one suffices all requirements noted Section 2 and power is enough low to cool.

Table 1: New PMT base Performance

\begin{tabular}{ccc}
\hline item & value & note \\
\hline Power consumption & $150 \mathrm{~mW}$ & $\mathrm{KTeV}$ base was $700 \mathrm{~mW}$ \\
Signal level & $1.15 \mathrm{mV}_{\mathrm{p}-\mathrm{p}} / \mathrm{MeV}$ & \\
Noise level & $180 \mu \mathrm{V}_{\mathrm{rms}}$ & \\
Charge equivalent gain & $3.3 \times 10^{5}$ & $\mathrm{KTeV}$ base was 5000 \\
Linearity & less than $5 \%$ & $1 \mathrm{MeV}$ to $1.3 \mathrm{GeV}$ \\
rate stability & less than $5 \%$ & 0.1 to $500 \mathrm{kHz}$ at $700 \mathrm{MeV}$ \\
\hline
\end{tabular}

\section{References}

[1] Federico Mescia and Christopher Smith, Phys. Rev. D, Vol. 76, No. 3, 034017, 2007.

[2] Takeo Inami and C. S. Lim, Prog. Theor. Phys., Vol. 65, No. 5, p.1772, 1981.

[3] Marciano, William J. and Parsa, Zohreh, Phys. Rev. D, Vol. 53, No. 1, R1, 1996.

[4] J. D. Cockcroft and E. T. S. Walton, Proc. of Soc. London, Vol. 137, No. 831, p. 229, 1932. 